Supplemental appendix for

\title{
The antifungal effect of piezoelectric charges on PMMA dentures
}

Carolina Montoya ${ }^{1}$, Julia Kurylec ${ }^{1}$, Divyashri Baraniya ${ }^{1}$, Aparna Tripathi ${ }^{1}$, Sumant Puri ${ }^{1, *}$, Santiago Orrego ${ }^{1,2, *}$

${ }^{1}$ Department of Oral Health Sciences, Kornberg School of Dentistry, Temple University. Philadelphia, PA.

${ }^{2}$ Bioengineering Department, College of Engineering, Temple University. Philadelphia, PA.

*Correspondence:

Prof. Santiago Orrego

Temple University

Department of Oral Health Sciences

Bioengineering Department

3223 North Broad Street. AEGD Clinic - Room 2E15. Philadelphia, PA. 19140.

sorrego@temple.edu

$+1-215-707-3817$

Sumant Puri

Temple University

Department of Oral Health Sciences

3223 North Broad Street. L215. Philadelphia, PA. 19140.

sumantpuri@temple.edu

$+1-215-707-5984$ 
This document includes:

Appendix 1: Roughness measurements

Appendix 2: Electrical poling and electromechanical characterization

Appendix 3: Contact angle measurements

Appendix 4: Effect of cyclic loading on PMMA composites surface

Appendix 5: Fluorescence microscopy of C. albicans biofilms

Appendix 6: Primer sequences used for RT-qPCR.

Appendix 7: Influence of filler quantity on the mechanical and electromechanical properties of PMMA composites

Appendix 8: Effect of electrical charge on the metabolic activity of $C$. albicans biofilms

Appendix 9: Antifungal effect of piezoelectric charges on Bis-GMA/TEGDMA piezoelectric dental composites 


\section{Appendix 1: Roughness measurements}

Surface roughness can modulate the adhesion and growth of $C$. albicans biofilms. ${ }^{1}$ An increase in surface roughness above $0.2 \mu \mathrm{m}$ causes increased microbial colonization and biofilm accumulation. ${ }^{2}$ To avoid the effects of surface roughness in biofilm formation, samples were polished incrementally from 400 to 2000 grit after complete polymerization. Then, a contact profilometer (Surfcorder SE 1700) was used to measure the mean roughness. Each sample was measured in 5 randomly selected locations. Only samples with an average roughness lower than $0.2 \mu \mathrm{m}$ were used in the following evaluations. The mean surface roughness for all samples (See. appendix Fig. S1) was lower than $0.2 \mu \mathrm{m}$, with a significantly higher value for the piezoelectric PMMA compared to the control group.

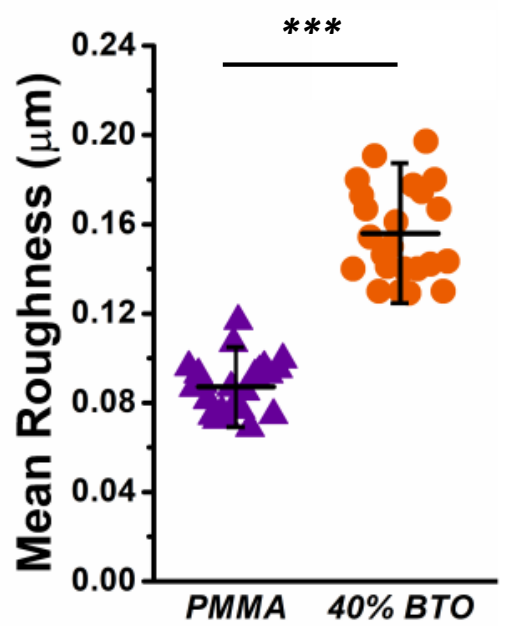

Appendix Figure S1. Mean surface roughness ( $\mathrm{Ra}$ ) for PMMA and PMMA piezoelectric composites after polishing. For each sample, 5 measurements were made. The surface roughness of all samples was lower than $0.2 \mu \mathrm{m}$. Significant differences between groups are expressed as $* * * \mathrm{p}<0.0001$.

\section{Appendix 2: Electrical poling and electromechanical characterization}

The piezoelectric performance of the PMMA filled with BT nanoparticles was evaluated using the Berlincourt method. ${ }^{3}$ Rectangular beams of $5 \times 1 \times 18 \mathrm{~mm}^{3}$ were prepared ( $\mathrm{N}=5$ per group). Before electromechanical characterization (electrical charge generation), samples were subjected to a high electric field $\left(20 \mathrm{kV} / \mathrm{mm}\right.$ ) at $140^{\circ} \mathrm{C}$ for $40 \mathrm{~min}$ (poling) ${ }^{4}$ and then stored in distilled water for $24 \mathrm{~h}$ at $37^{\circ} \mathrm{C}$.

Then, electrodes of conductive carbon tape with an area of $40 \mathrm{~mm}^{2}$ were adhered to the top and bottom surfaces of the beam. The electrodes were connected to a piezo amplifier (TE Connectivity Piezo film lab amplifier), which amplifies the charge signal. The preamplifier was connected to a data acquisition system DAQ (National Instruments USB-6008), which was connected to the computer via USB. An actuator (TA Instruments ElectroForce 5500) was programmed to apply a load of $5 \mathrm{~N}$ (which resembles mastication stresses $\sim 22 \mathrm{MPa}^{5}$ ) following a sinusoidal waveform with $2 \mathrm{~Hz}$ frequency. This loading frequency was selected because it resembles the natural chewing activity of humans. ${ }^{6}$ The analog signal of the load cell and the preamplifier were sent to the computer. A program was written in LabVIEW (National Instruments 
v.2018) to record both signals. The effect of the poling process on the charge generation of the PMMA composite filled with $40 \%$ of BT is presented in appendix Fig. S2. A significant increase in the electrical charge generated by the composite after the poling process was measured when compared with the same material without being poled.

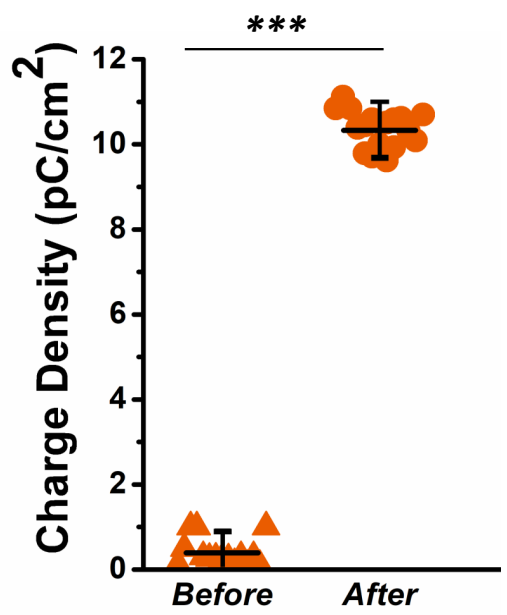

Appendix Figure S2. Effect of electrical poling on the electrical charge generation of the PMMA composite filled with $40 \%$ of BT. The standard deviation bars were obtained from $\mathrm{N}=7$ samples. Significant differences between groups are expressed as $* * * p<0.0001$.

\section{Appendix 3: Contact angle measurements}

The wettability of biomaterial surfaces can influence biofilm formation and can play an important role in the adhesion of microorganisms. ${ }^{7,8}$ The contact angle of both the PMMA and PMMA piezoelectric composites after poling was measured by placing $10 \mu \mathrm{L}$ of ultrapure water on the sample surfaces. A photograph of each droplet was captured immediately after deposition. The contact angle was measured using the ImageJ software. Five $(\mathrm{N}=5)$ samples were evaluated for each polymer group. As presented in appendix Figure S3, the contact angle for both groups (PMMA and PMMA piezo-composite) is statistically similar $\left(\sim 69^{\circ}\right)$. These values are consistent with previous reports for the contact angle of PMMA. ${ }^{9}$ 


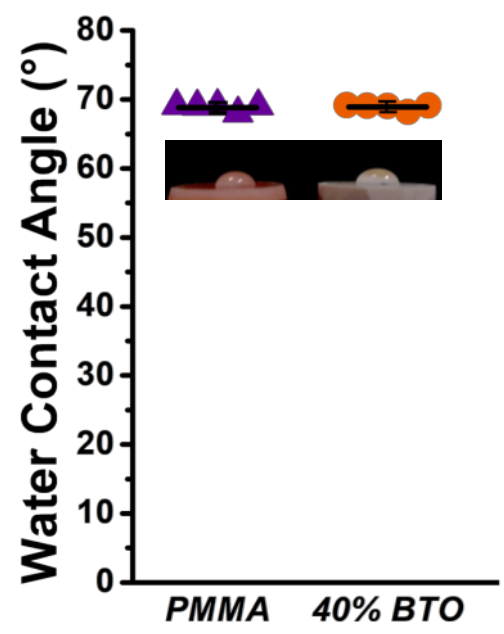

Appendix Figure S3. Water contact angle measurements for PMMA and PMMA piezoelectric composites. The error bars were obtained from $\mathrm{N}=5$ measurements. No statistically significant differences were found between groups $(p=0.745)$.

\section{Appendix 4: Effect of cyclic loading on the PMMA composite surface}

To evaluate the potential influence of repetitive loading on the morphology of the composite surface, we analyzed the surfaces of PMMA samples filled with $40 \%$ of BTO before and after being mechanically loaded at compression $(-0.5 \mathrm{~N}$ to $5 \mathrm{~N}$ and $2 \mathrm{~Hz})$ continuously for $24 \mathrm{~h}$. A scanning electron microscope (FEI Quanta $450 \mathrm{EFG}$ ) was used to observe the surface of the composite at an accelerating voltage of $10 \mathrm{kV}$. As shown in Fig S4, the BT nanoparticles can be seen distributed along the PMMA surface. No evident changes (i.e., cracks, indents, porosity, or changes in roughness) were observed along the samples surfaces before and after the continuous cyclic stimulation.

\section{a) Before Cyclic Mechanical Loading}

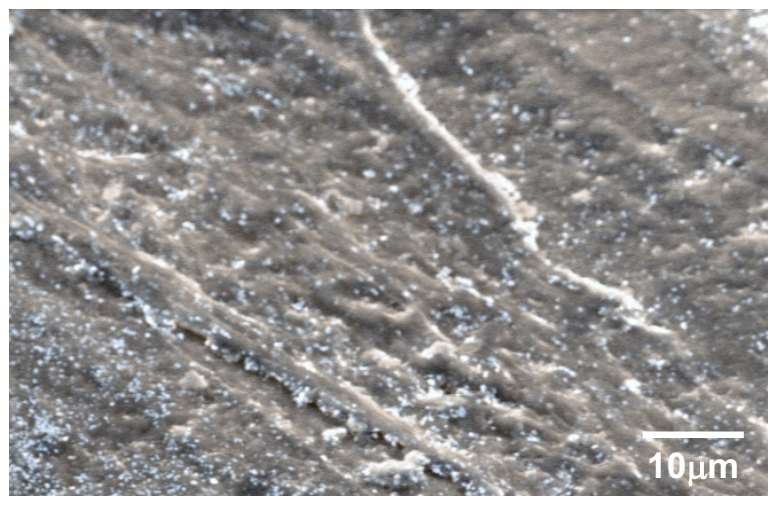

b) After Cyclic Mechanical Loading

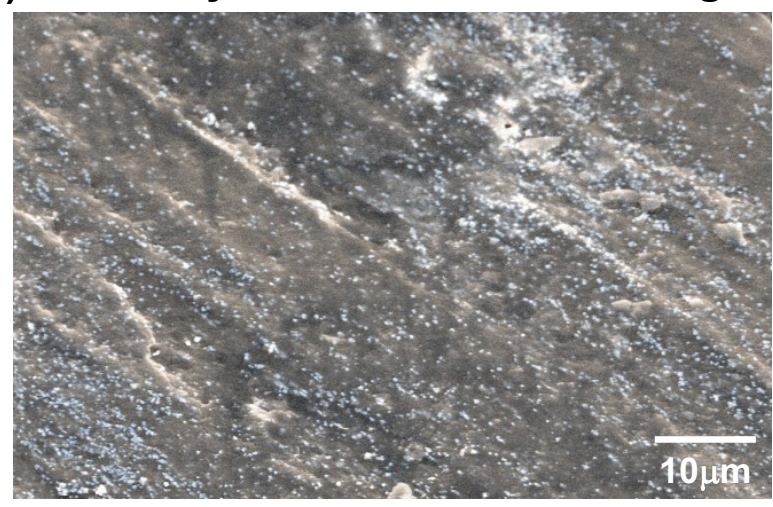

Appendix Figure S4. Scanning electron micrograph of PMMA samples filled with $40 \%$ of BT. a) Before cyclic mechanical stimulation, b) After $24 \mathrm{~h}$ of cyclic mechanical loading at $5 \mathrm{~N}$ and $2 \mathrm{~Hz}$. 


\section{Appendix 5: Fluorescence microscopy of $C$. albicans biofilms}

To visualize and quantify the living and the dead bacteria on top of the samples, the biofilm was stained with a SYTO9/propidium iodide (PI) solution. The fluorescent SYTO9 stains live yeasts with intact cell membranes, whereas dead yeasts with damaged membranes are stained with the PI. The solution stain was prepared by mixing $3 \mathrm{~mL}$ of SYTO9 and $3 \mathrm{~mL}$ of propidium iodide (PI) in $1 \mathrm{~mL}$ of ultrapure water. ${ }^{10}$ On the surface of each sample, $200 \mathrm{~mL}$ of the staining solution was applied and incubated in the dark for $20 \mathrm{~min}$. Finally, samples were gently rinsed with filtersterilized water to remove the excess of dye.

To quantify the proportion of live and dead yeast, the color images (appendix Fig. S5) were converted to grayscale images with pixel values from 0 to 255 . Then, a similar threshold was applied to obtain black and white images. All pixels with values above the threshold (white pixels) were counted. The number of white pixels was computed separately for both live and dead images. The proportion of live and dead cells are presented in Fig. 2 of the main document.

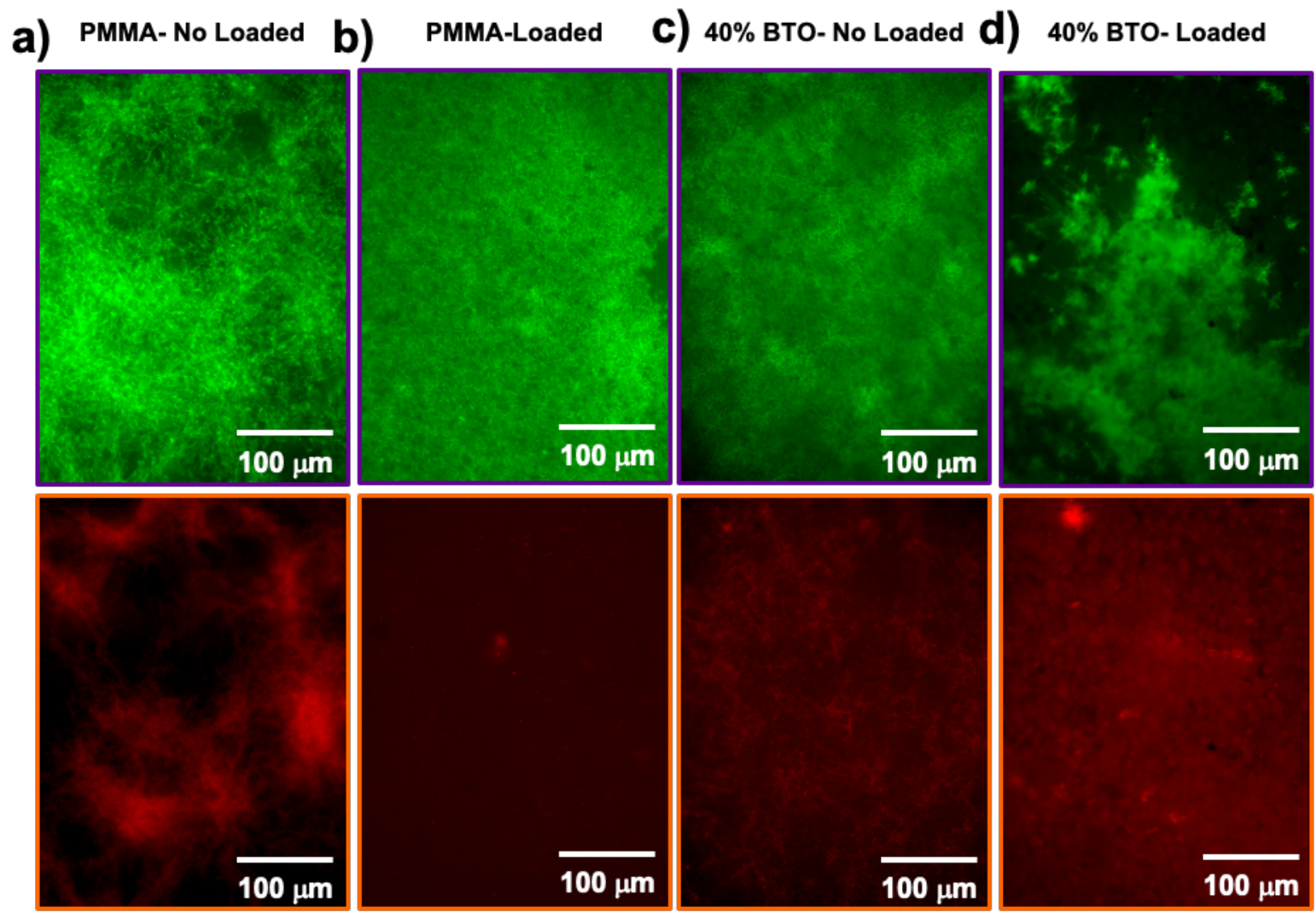

Appendix Figure S5. LIVE/DEAD staining of $C$. albicans biofilms cultivated under different materials and conditions. a) PMMA no loaded, b) PMMA under mechanical stimulation, c) PMMA filled with $40 \%$ of BT no loaded and d) BT composites under mechanical stimulation. Live yeasts with intact cell membranes are stained green, whereas dead yeasts with damaged membranes are stained red.

Appendix 6: Primer sequences used for RT-qPCR. 
Appendix Table S6. List of primer sequences used for RT-PCR analysis in this study.

F: Forward primer. R: reverse primer.

\begin{tabular}{|c|l|}
\hline Gene & \multicolumn{1}{c|}{ Primer Sequences } \\
\hline \multirow{2}{*}{$18 s r R N A$} & $\begin{array}{l}\text { F: 5'-GGATTTACTGAAGACTAACTACTG-3' } \\
\text { R: 5'-GAACAACAACCGATCCCTAGT-3' }\end{array}$ \\
\hline \multirow{2}{*}{ ALS1 } & $\begin{array}{l}\text { F: 5'-GACTAGTGAACCAACAAATACCAG-3' } \\
\text { R: 5'- ACCAGAAGAAACAGCAGGTG-3' }\end{array}$ \\
\hline \multirow{2}{*}{$H W P 1$} & $\begin{array}{l}\text { F: 5'-GCTACCACTTCAGAATCATCATC-3' } \\
\text { R: 5'-GCACCTTCAGTCGTAGAGACG-3' }\end{array}$ \\
\hline \multirow{2}{*}{ GSC1 } & $\begin{array}{l}\text { F: 5'-GAAATCGGCATATGCTGTGTC-3' } \\
\text { R: 5'-ATGAACGACCAATGGAGAAG-3' }\end{array}$ \\
\hline \multirow{2}{*}{ SOD5 } & F: 5'-ACGAGGGACACGGCAATGCT-3' \\
& R: 5'-GCGCCATTACCTTGAGGAGCAGTA-3' \\
\hline
\end{tabular}

\section{Appendix 7: Influence of filler quantity on the mechanical and electromechanical properties of PMMA composites}

To study the effect of BT quantity on the mechanical properties of the PMMA composites, samples (N=4-6 per group) with varying quantities of BT (10, 20, 40 wt.\%) were prepared following the protocol presented in Section 2.1 of the main document. After preparation, samples were stored in purified water at $37^{\circ} \mathrm{C}$ for $24 \mathrm{~h}$. The mechanical properties were determined using a three-point bending configuration following the ISO 20795-1 for denture materials. Flexural strength $(\sigma)$ and modulus $(E)$ were calculated. As seen in Fig. S7-1, no significant changes were found on the flexural strength of composites with fillers up to $20 \%(\sim 65 \mathrm{MPa})(p>0.05)$. A significant reduction in the strength was measured for the $40 \%$ samples $(\sim 50 \mathrm{MPa})(p<0.05)$. No significant changes were observed on the flexural modulus by varying the filler quantity.
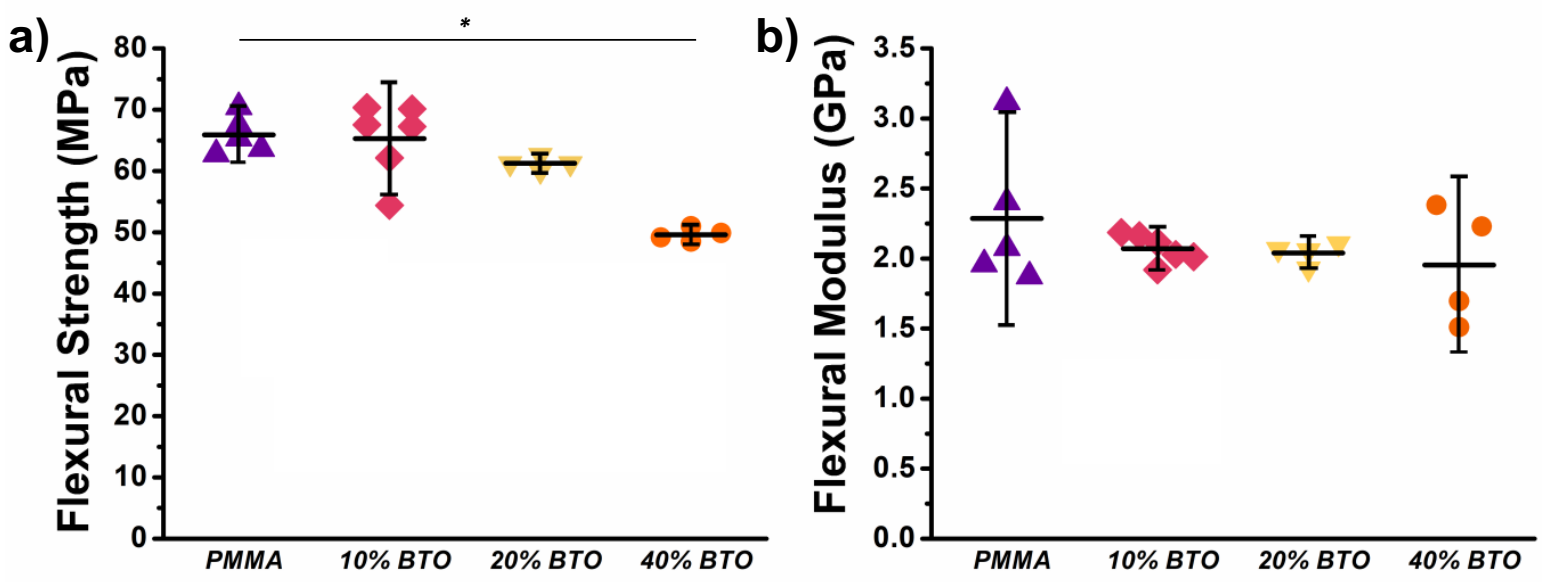

Figure S7-1. Effect of the BT content on the mechanical properties of PMMA composites. a) Flexural strength, b) Flexural modulus. The error bars were obtained from $\mathrm{N}=4-6$ samples for each group. Significant differences between groups are expressed as follows: ${ }^{*} p<0.05,{ }^{*} p<0.001$ or $* * * p<0.0001$. 
The electromechanical properties of the PMMA composites were measured as explained in Appendix 2. As expected, no electrical charge was measured for the PMMA without BT fillers, while for the PMMA composites, the generated electrical charge was proportional to the amount of piezoelectric filler.

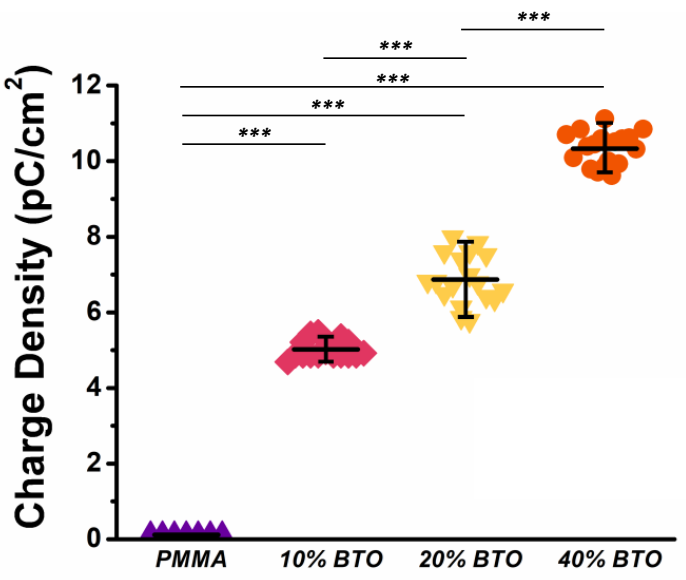

Figure S7-2. Effect of the BT content on the electrical charge density (charge generation) of PMMA composites after being subjected to a three-point bending cyclic load of $5 \mathrm{~N}$ at $2 \mathrm{~Hz}$. The error bars were obtained from $\mathrm{N}=4$ samples for each group. Significant differences between groups are expressed as follows: ${ }^{*} p<0.05,{ }^{* *} p<0.001$ or ${ }^{* * *} p<0.0001$

\section{Appendix 8: Effect of electrical charge on the metabolic activity of $C$. albicans biofilms}

To study the effect of different electrical charges (different magnitudes of mechanical load) on biofilm formation, we evaluated the metabolic activity of biofilms cultivated under different conditions. C. albicans biofilms were cultivated as explained in the main document (sec. 2.3) on the surface of PMMA composites filled with $40 \%$ on BT. After the 3-hour adhesion period, samples were incubated for $24 \mathrm{~h}$ applying different mechanical loads that render three different electrical charges $(1 \mathrm{pC}, 8.5 \mathrm{pC}$ and $10.5 \mathrm{pC})$. After the 24- hour incubation, biofilms were washed with PBS to detach non-adherent cells and the metabolic activity of the biofilms was measured via MTT. As presented in appendix Fig. S8, there is a change in the metabolic activity of the cells with the electrical charge. An increase in the electrical charge seems to produce a higher reduction in the biofilm activity. 


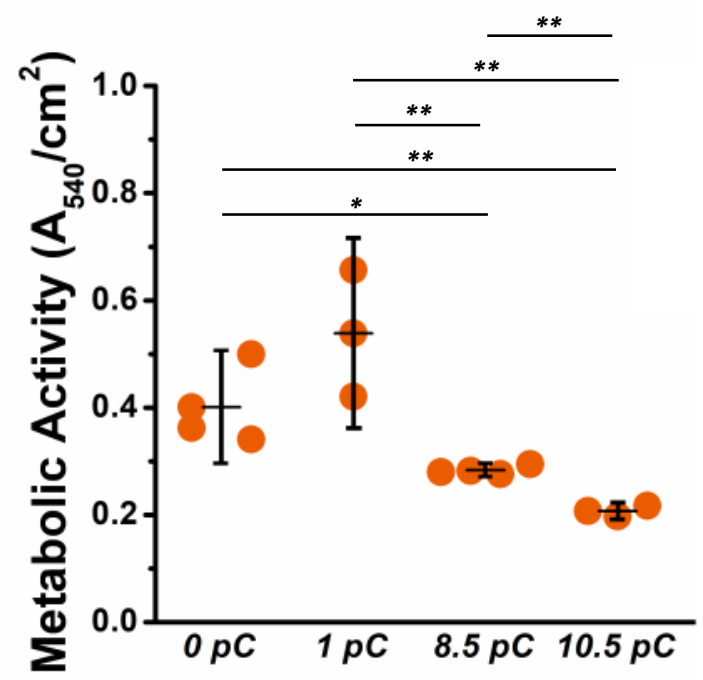

Appendix Figure S8. Metabolic activity of $C$. albicans biofilms cultivated on the surface of piezoelectric composite filled with $40 \%$ of BT subjected to different mechanical loads (electrical charge generated). For all tests, the error bars were obtained from $\mathrm{N}=4$ measurements. Significant differences between groups are expressed as follows: ${ }^{*} p<0.05,{ }^{*} p<0.001$ or $* * * p<0.0001$.

\section{Appendix 9: Antifungal effect of piezoelectric charges on Bis-GMA/TEGDMA piezoelectric dental composites}

To study the antifungal effect of piezoelectric charges on other dental materials, we evaluated the $C$. albicans biofilm formation on Bis-GMA/TEGDMA dental composites filled with BT nanoparticles. We prepared piezoelectric resin composites according to Reference \#31 from the main document. Briefly, samples were fabricated by mixing bisphenol A diglycidyl dimethacrylate (Bis-GMA) (Sigma 494356) and triethylene glycol dimethacrylate (TEGDMA) (Sigma 261548) mixed in a 1:1 ratio by weight; camphorquinone (CQ) (Sigma 124893) and ethyl 4dimethylaminobenzoate (4E) (Sigma E24905) were added as the initiator and co-initiator in proportions of 0.2 and $0.8 \mathrm{wt} \%$ of the resin, respectively. The blend was mixed with $10 \%(\mathrm{wt} / \mathrm{wt})$ of BT nanoparticles (US Nanomaterials US3830, $200 \mathrm{~nm}$ ) and 60\% (wt/wt) of silanized barium boroaluminosilicate glass fillers (median diameter of $1.4 \mu \mathrm{m}$, Dentsply Caulk). Mixing was conducted using a planetary mixer (Thinky ARE-310) for $3 \mathrm{~min}$ at $2000 \mathrm{rpm}$. The liquid mixture was then poured into a mold and light-cured with a LED unit (Cure TC-3, Spring Health Products). The mold's top and bottom surfaces were irradiated for one minute on each side, resulting in a total radiant exposure of $166 \mathrm{~J} / \mathrm{cm}^{2}$ (equivalent to $1 \mathrm{~min}$ at $980 \mathrm{~mW} / \mathrm{cm}^{2}$ ). After curing, the roughness of the surfaces was verified as $<0.2 \mu \mathrm{m}$, and the samples were stored in distilled water for 24 hours at $37^{\circ} \mathrm{C}$ for the release of unreacted monomers. 
C. albicans biofilms were cultivated on the Bis-GMA/TEGDMA piezoelectric dental composites, as explained in the main document (Section 2.3). After the 3-hour adhesion period, samples were incubated for $24 \mathrm{~h}$ applying a continuous cyclic mechanical load that rendered an electrical charge of $1.2 \mathrm{pC}$. After the 24- hour incubation, biofilms were washed with PBS to detach non-adherent cells and the metabolic activity of the biofilms was measured via MTT. As presented in appendix Fig. S9, there is a decrease in the metabolic activity of the C. albicans cells after activation of the piezoelectric charges. A reduction of $30 \%$ in the metabolic activity was measured compared to no stimulated samples (no activation of the piezoelectric charges). This reduction in the metabolic activity on Bis-GMA/TEGDMA piezoelectric dental composites shows that the antifungal effect of piezoelectric charges can be translated to other material matrixes.

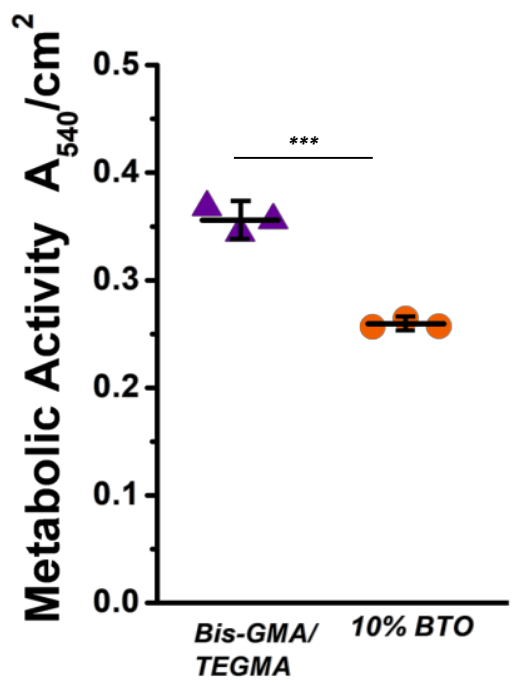

Appendix Figure S9. Metabolic activity of $C$. albicans biofilms cultivated on piezoelectric composites fabricated with Bis-GMA/TEGDMA matrix and 10\% of BT filler. Samples were stimulated with a mechanical load that renders $1.2 \mathrm{pC}$ of electrical charge. For all tests, the error bars were obtained from $\mathrm{N}=3$ measurements. Significant differences between groups are expressed as follows: ${ }^{*} p<0.05,{ }^{* *} p<0.001$ or ${ }^{* * *} p<0.0001$. 


\section{References}

1. Pereira-Cenci, T.; Del Bel Cury, A. A.; Crielaard, W.; Ten Cate, J. M., Development of Candida-Asociated Denture Stomatitis: New Insights. J Appl Oral Sci 2008, 16 (2), 86-94.

2. Zissis, A. J.; Polyzois, G. L.; Yannikakis, S. A.; Harrison, A., Roughness of Denture Materials: A Comparative Study. Int J Prosthodont 2000, 13 (2), 136-40.

3. Fialka, J.; Beneš, P., Comparison of Methods of Piezoelectric Coefficient Measurement. In 2012 IEEE International Instrumentation and Measurement Technology Conference Proceedings, IEEE: 2012; pp 37-42.

4. Waller, D.; Safari, A., Corona Poling of PZT Ceramics and Flexible Piezoelectric Composites. Ferroelectrics 1988, 87 (1), 189-195.

5. Sadr, K.; Alipour, J.; Heidary, F., Finite Element Analysis of Soft-lined Mandibular Complete Denture and its Supporting Structures. Journal of dental research, dental clinics, dental prospects 2012, 6 (2), 37.

6. Po, J.; Kieser, J.; Gallo, L. M.; Tésenyi, A.; Herbison, P.; Farella, M., Time-frequency Analysis of Chewing Activity in the Natural Environment. Journal of dental research 2011, 90 (10), 1206-1210.

7. Ista, L. K.; Mendez, S.; Lopez, G. P., Attachment and Detachment of Bacteria on Surfaces with Tunable and Switchable Wettability. In Biofouling, England, 2010; Vol. 26, pp 111-8.

8. Zheng, S.; Bawazir, M.; Dhall, A.; Kim, H.-E.; He, L.; Heo, J.; Hwang, G., Implication of Surface Properties, Bacterial Motility, and Hydrodynamic Conditions on Bacterial Surface Sensing and Their Initial Adhesion. Frontiers in Bioengineering and Biotechnology 2021, 9 (82). 9. Ma, Y.; Cao, X.; Feng, X.; Ma, Y.; Zou, H., Fabrication of Super-hydrophobic Film from PMMA with Intrinsic Water Contact Angle below 90. Polymer 2007, 48 (26), 7455-7460.

10. Robertson, J.; McGoverin, C.; Vanholsbeeck, F.; Swift, S., Optimisation of the Protocol for the LIVE/DEAD ${ }^{\circledR}$ BacLightTM Bacterial Viability Kit for Rapid Determination of Bacterial Load. Frontiers in Microbiology 2019, 10 (801). 